\title{
Acinetobacter calcoaceticus/baumannii Complex
}

National Cancer Institute

\section{Source}

National Cancer Institute. Acinetobacter calcoaceticus/baumannii Complex. NCI

Thesaurus. Code C86091.

A non taxonomic grouping of bacteria assigned to the Acinetobacter genus that include the A. calcoaceticus and A. baumannii species. 